\title{
Two or three insulin injections in adolescence?
}

\author{
F R J HINDE AND D I JOHNSTON
}

Children's Department, University Hospital, Queen's Medical Centre, Nottingham

SUMMARY Morning hyperglycaemia remains a challenge to conventional insulin regimens. Eighteen adolescents participated in a one year crossover study to examine the effect of delaying the evening intermediate acting insulin from before the evening meal to bedtime. This three injection regimen caused slightly higher blood glucose concentrations in the early part of the night, and lower concentrations in the morning, but no overall change in glycosylated haemoglobin concentrations $\left(\mathrm{HbA}_{1 \mathrm{c}}\right)$. Seasonal change accounted for substantially more of the variance in $\mathrm{HbA}_{1 \mathrm{c}}$ concentrations than did the regimen change. The three injection regimen did not alter the frequency of hypoglycaemic episodes.

Metabolic control on both regimens might have been improved by more intensive monitoring and medical attention. This study suggests that factors beyond medical control, such as seasonal variation, may contribute more to the control of diabetes in adolescents than changes in conventional insulin regimens, particularly when unaccompanied by intensive monitoring.

The classic primary aims of treatment for the child with insulin dependent diabetes are to maintain normal growth and development while preventing symptomatic hypo- or hyperglycaemia. ${ }^{1}$ As the evidence relating glycaemic control to microvascular complications has increased, ${ }^{2}$ so the emphasis of management has been extended towards achieving normoglycaemia. ${ }^{3}$

One of the major barriers to glycaemic control on conventional insulin regimens is the dawn phenomenon. This consists of a rise in blood glucose concentrations that starts in the early hours of the morning and continues after breakfast, causing a mid-morning glycaemic peak. ${ }^{4}$ Although the dawn phenomenon has been shown to be largely due to the glycaemic effects of nocturnal secretion of growth hormone,${ }^{5}$ it is likely to be exacerbated by the failure of conventional insulin regimens to maintain insulin concentrations throughout the night. Attempts to prevent morning hyperglycaemia by increasing the evening insulin dose may result in nocturnal hypoglycaemia, and the ensuing 'Somogyi effect' will further compromise blood glucose control. ${ }^{6}$ Nocturnal hypoglycaemia thus limits the effectiveness of twice daily insulin regimens in controlling the dawn phenomenon.

Delaying the intermediate acting portion of the evening insulin injection from before the evening meal until bedtime has been shown to improve morning blood glucose concentrations, without in- creasing nocturnal hypoglycaemia, in adult patients selected because they showed a noticeable dawn phenomenon. ${ }^{7}$ The incidence and severity of this phenomenon in childhood are not known. We have therefore performed a one year crossover study comparing the classic twice daily 'mixed' injection with a three injection regimen involving a split evening injection. The study was performed on adolescent patients because we hoped that this older age group would be more tolerant of the third injection; the notorious poor control at this time also suggested significant possible benefits. ${ }^{8}$ Children were not selected on the grounds of glycaemic control, as we wished to establish the value of this regimen, and thus the importance of the dawn phenomenon, to the 'average' adolescent patient.

\section{Methods}

Patients. Nineteen patients were selected from the population attending the Nottingham Children's Diabetic Clinic on the grounds of age (adolescent) and duration of diabetes ( $>2$ years) (Table 1). All children and their parents gave informed consent to the trial, which had the approval of the local ethical committee. One girl failed to comply with the trial protocol and was, therefore, excluded. Results presented are from the 18 patients who completed the trial, none of whom suffered any other major illness during the study period. 
Table 1 Patient details at randomisation

\begin{tabular}{|c|c|c|c|c|c|c|}
\hline $\begin{array}{l}\text { Case } \\
\text { No }\end{array}$ & Sex & $\begin{array}{l}\text { Age } \\
\text { (decimal } \\
\text { years) }\end{array}$ & $\begin{array}{l}\text { Duration of } \\
\text { diabetes } \\
\text { (decimal } \\
\text { years) }\end{array}$ & $\begin{array}{l}H b A_{l c} \\
(\%)\end{array}$ & $\begin{array}{l}\text { Insulin } \\
\text { dose } \\
\text { (ulkg/day) }\end{array}$ & $\begin{array}{l}\text { Post prandial } \\
\text { C peptide* } \\
\text { (nmol/l) }\end{array}$ \\
\hline 1 & $\mathbf{M}$ & $13 \cdot 4$ & $6 \cdot 8$ & $9 \cdot 8$ & 0.87 & 0.01 \\
\hline 2 & $\mathbf{M}$ & $15 \cdot 5$ & $10 \cdot 7$ & $13 \cdot 7$ & 0.90 & $0 \cdot 02$ \\
\hline 3 & $\mathrm{~F}$ & $15 \cdot 7$ & $13 \cdot 7$ & $11 \cdot 1$ & 1.08 & 0.03 \\
\hline 4 & $\mathrm{~F}$ & $14 \cdot 6$ & $6 \cdot 6$ & $13 \cdot 1$ & 0.99 & 0.02 \\
\hline 6 & $\mathrm{~F}$ & $15 \cdot 1$ & $4 \cdot 6$ & $11 \cdot 7$ & $1 \cdot 10$ & 0.03 \\
\hline 7 & $\mathrm{~F}$ & $14 \cdot 5$ & $6 \cdot 1$ & $12 \cdot 1$ & 1.04 & 0.02 \\
\hline 8 & $\mathbf{M}$ & $12 \cdot 7$ & $12 \cdot 0$ & $10 \cdot 0$ & 0.90 & 0.01 \\
\hline 9 & $\mathbf{M}$ & $14 \cdot 4$ & $10 \cdot 7$ & $10 \cdot 9$ & 1.07 & 0.01 \\
\hline 10 & $\mathrm{~F}$ & $15 \cdot 2$ & $3 \cdot 2$ & $12 \cdot 7$ & 0.96 & 0.02 \\
\hline 11 & $\mathrm{~F}$ & $10 \cdot 8$ & $2 \cdot 5$ & $12 \cdot 6$ & 1.24 & 0.03 \\
\hline 12 & $\mathrm{~F}$ & 15.8 & $11 \cdot 0$ & $12 \cdot 6$ & 0.77 & 0.01 \\
\hline 13 & $\mathrm{~F}$ & $12 \cdot 2$ & $2 \cdot 3$ & 10.9 & $1 \cdot 12$ & 0.16 \\
\hline 14 & $\mathrm{~F}$ & $12 \cdot 0$ & $7 \cdot 4$ & $11 \cdot 8$ & 1.26 & 0.01 \\
\hline 17 & $\mathrm{M}$ & $15 \cdot 8$ & $\begin{array}{r}0.1 \\
14 \cdot 2\end{array}$ & 13.9 & 1.37 & 0.02 \\
\hline 18 & $\mathbf{M}$ & $14 \cdot 8$ & $2 \cdot 2$ & $11 \cdot 1$ & 0.76 & 0.02 \\
\hline Mean & & $14 \cdot 2$ & $7 \cdot 4$ & $12 \cdot 4$ & 1.05 & \\
\hline SD & & 1.5 & 3.9 & $2 \cdot 0$ & 0.17 & \\
\hline Range & & $(10 \cdot 8-15 \cdot 9)$ & $(2 \cdot 2-14 \cdot 2)$ & $(9 \cdot 8-18 \cdot 1)$ & $(0 \cdot 76-1 \cdot 37)$ & \\
\hline
\end{tabular}

${ }^{*}$ Adult fasting reference range $0 \cdot 18-0.52 \mathrm{nmol} / \mathrm{l}$.

All children had normal renal function and were normotensive with no clinical evidence of microvascular complications. Only one child showed significant endogenous insulin production as shown by measurements of post prandial $\mathrm{C}$ peptide concentrations. Their mean concentration of glycosylated haemoglobin $\left(\mathrm{HbA}_{1 \mathrm{c}}\right)$ at randomisation $(12.4 \%)$ was slightly higher than the clinic age group mean $(11.8 \%)$. At entry to the study all children were being treated with injections of mixed intermediate and short acting highly purified porcine insulin, given before breakfast and before the main evening meal-a two injection regimen.

Procedure. After a four month run in period the children were randomly allocated to two groups. One group continued the two injection regimen. The other group received the trial three injection regimen: a normal 'mixed' morning injection, an injection of short acting insulin only given before the main evening meal, and an injection of intermediate acting insulin shortly before bed. After four months the children were switched over to the alternative regimen for the final four months of the study. The last child to be randomised entered and completed the study 11 weeks after the first. The total daily insulin dose, as well as the proportions taken as morning or evening, short or intermediate, acting insulin were similar on both regimens (Table 2).

Table 2 Anthropometry, hypoglycaemia, and insulin dose in the patients when given two or three injections. All values are mean $(S E)$

\begin{tabular}{lll}
\hline & Patients given two injections & Patients given three injections \\
\hline Height gain (cm/four months) & $1 \cdot 39(0 \cdot 27)$ & $1 \cdot 31(0 \cdot 25)$ \\
Weight gain (kg/four months) & $1 \cdot 02(0 \cdot 52)$ & $1 \cdot 90(0 \cdot 42)$ \\
Hypoglycaemic episodes: & $4 \cdot 1(1 \cdot 2)$ & $3 \cdot 9(0 \cdot 91)$ \\
mild & $0 \cdot 4(0 \cdot 1)$ & $0 \cdot 2(0 \cdot 2)$ \\
moderate & 0 & $0 \cdot 1(0 \cdot 1)$ \\
severe & $1 \cdot 03(0 \cdot 04)$ & $1 \cdot(06(0 \cdot 04)$ \\
Total insulin dose (U/kg) & $22 \cdot 9(1 \cdot 3)$ & $22 \cdot 6(1 \cdot 3)$ \\
$\%$ Total insulin doses: & $40 \cdot 1(1 \cdot 5)$ & $39 \cdot 6(1 \cdot 7)$ \\
morning short acting & $12 \cdot 0(1 \cdot 0)$ & $13 \cdot 5(0 \cdot 9)$ \\
morning intermediate acting & $24 \cdot 9(2 \cdot 0)$ & $24 \cdot 3(1 \cdot 7)$ \\
evening short acting & & \\
evening intermediate acting &
\end{tabular}

$\mathrm{p}=$ Not significant in all cases (performed by paired $t$ test). 
Children were seen at monthly intervals throughout the study, clinic attendances taking place at randomisation and at the end of both study periods. All other consultations took place in the children's homes. During the run in period efforts were made to improve metabolic control, with particular emphasis on reducing morning hyperglycaemia while attempting to avoid nocturnal hypoglycaemia.

Monitoring. At each consultation details of insulin dosage, diabetic symptoms, and intercurrent illnesses were recorded, and a capillary blood specimen obtained for estimation of $\mathrm{HbA}_{1 \mathrm{c}}$ concentrations. Children were asked to provide a four point blood glucose series (three pre prandial and one bedtime measurement) twice weekly, with midnight and 0300 measurements twice a month. Two methods of home blood glucose measurement were used: five patients used Dextrostix (Ames) read with Glucochek meters (Medistron Limited); the remainder used visually read BM 20/800 sticks (BoehringerMannheim). The method of measurement remained constant for each child throughout the study. During the three days before each consultation children used Sarstedt fluoride capillary tubes to provide one eight point blood glucose series (before and after the three main meals, midnight, and 0300), and two additional fasting specimens. These tubes were stored at roughly $4^{\circ} \mathrm{C}$ until laboratory blood glucose analysis not more than four days later.

Height and weight were measured at each clinic visit, and a post prandial specimen of blood obtained on one occasion for $\mathrm{C}$ peptide assay and routine biochemistry.

Laboratory methods. Blood for assay of $\mathrm{HbA}_{1 \mathrm{c}}$ was taken into EDTA tubes; the cells were then washed in $0.85 \%$ saline, separated, and lysed in an acid haemolysing solution within two hours of sampling. The lysate was stored at $4^{\circ} \mathrm{C}$ until analysed by an electrophoretic method (Gellman Sciences Inc). ${ }^{9}$ Each specimen was analysed in duplicate, using different membranes; the means of the paired results are presented here. Blood for estimation of $\mathrm{C}$ peptide was taken into heparinised tubes containing Trasylol (Bayer), separated immediately, and the plasma stored at $-18^{\circ} \mathrm{C}$ until analysed by radioimmunoassay. ${ }^{10}$

Questionnaire. At the end of the study all patients completed a questionnaire comparing the two regimens. On a five point scale they indicated which regimen they considered had given best blood glucose control, made them feel generally better, caused more problems with hypoglycaemia, was more convenient, and which they would prefer to continue with.

\section{Results}

Blood glucose. During the two treatment periods children performed $85 \%$ of requested home and $88 \%$ of requested laboratory blood glucose measurements. Friedman two way analysis of variance showed that no consistent changes in blood glucose profiles took place within the period of either treatment regimen. The values at individual time points did not conform to a normal distribution, and the results were therefore assessed using the median values of each child's blood glucose concentrations at each time point. Comparisons between the regimens were made using Wilcoxon's matched pairs signed ranks test.

Both the home and laboratory blood glucose measurements showed only small differences between the regimens. The laboratory measurements (Fig. 1) showed that blood glucose concentrations were lower before breakfast on the three injection regimen $(p=0 \cdot 03)$. The home measured results

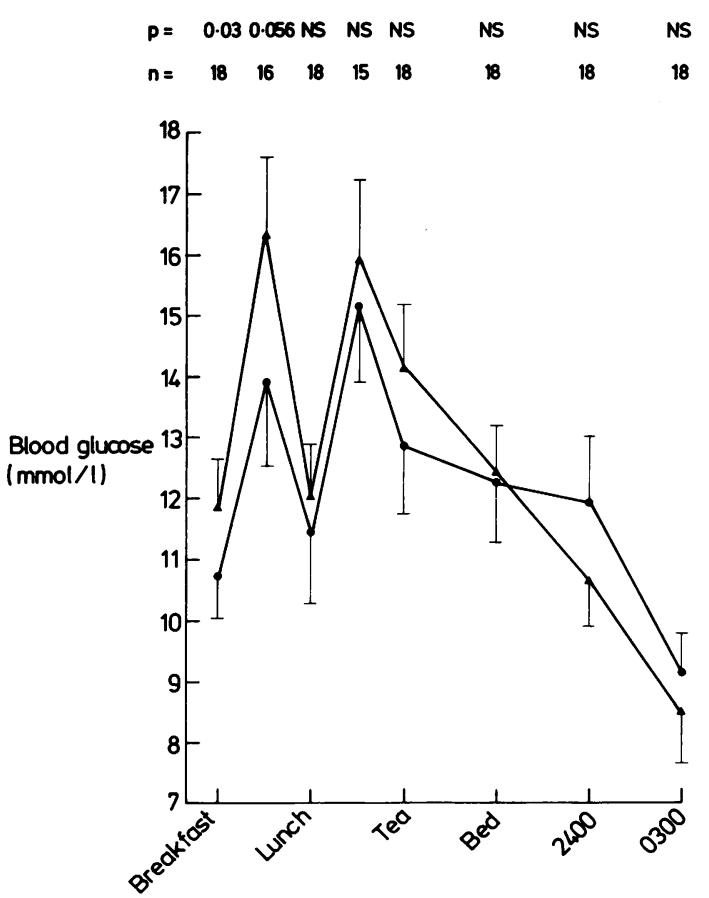

Fig. 1 Mean (SE) concentrations of laboratory measured blood glucose on two and three insulin injections a day ( $p$ values from Wilcoxon's matched pairs signed ranks test) ( $\Delta=$ two injections, $\mathbf{O}=$ three injections). 
showed a similar trend at this time. After breakfast blood glucose remained lower on this regimen, but this difference did not reach significance, possibly because only 16 children were able to provide sufficient samples for analysis at this time. Throughout the rest of the day blood glucose concentrations remained very similar on the two regimens, until 0300 , when the home measured results showed higher concentrations on three injections $(p<0.03)$. The laboratory measurements showed a similar trend at this time.

Hypoglycaemic episodes. (Table 2). These were recorded as: mild=mild symptoms rapidly relieved; moderate $=$ made the child unwell, caused an upset to family routine; and severe $=$ requiring admission to hospital, intravenous glucose, or glucagon. There was no difference between the regimens in any category of hypoglycaemia. Eight children experienced symptomatic nocturnal hypoglycaemia during the whole study; these episodes were slightly more common on two injections than three. Unrecognised nocturnal hypoglycaemia, defined as a home measured blood glucose concentration of less than $4.0 \mathrm{mmol} / \mathrm{l}(72 \mathrm{mg} / 100 \mathrm{ml})$, or a laboratory measured concentration of less than $3.5 \mathrm{mmol} / \mathrm{l}(63 \mathrm{mg} / 100 \mathrm{ml})$, occurred with equal frequency on both regimens. This occurred on about $10 \%$ of nights tested and affected all but two of the children during the year of the study.

Anthropometry (Table 2). The height and weight velocities on both regimens were similar.

Glycosylated haemoglobin $\left(\mathbf{H b A}_{\mathbf{1 c}}\right)$. The mean (SEM) values of each child's $\mathrm{HbA}_{1 \mathrm{c}}$ concentrations during the last two months of each treatment regimen were very similar $(11.67(0.46 \%)$ on two injections, 11.76 $(0.40 \%)$ on three injections). Individual children showed clear differences in control on the two regimens, but the direction and magnitude of these changes did not correlate with age, duration of diabetes, or with randomisation values of $\mathrm{HbA}_{1 \mathrm{c}}$ and fasting or mid-morning blood glucose. There was, however, considerable seasonal variation in $\mathrm{HbA}_{1 \mathrm{c}}$ concentrations (Fig. 2), the most prominent feature of which was a fall in mean concentrations during spring and early summer.

The seasonal variation was examined further with multiple regression analysis. This method was used in order to eliminate any effect of disproportionate patient representation in the monthly summaries. All $\mathrm{HbA}_{1 \mathrm{c}}$ concentrations from the whole study, including the run in period, were entered, together with identifiers of the child, month of sampling, and the regimen. 'Regimen' had three values: the run in

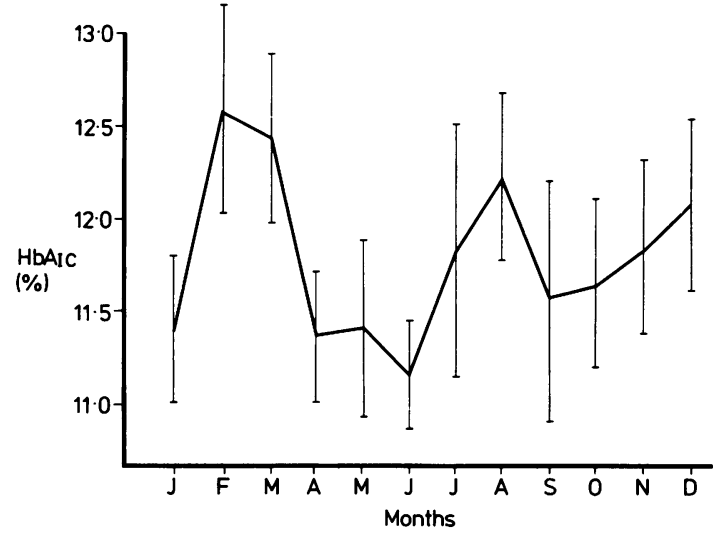

Fig. 2 Mean (SE) $\mathrm{HbA}_{\text {lc }}$ concentrations from the whole study, including the run in period, by month of sampling.

and both treatment periods. This showed that $60 \%$ of the variation in $\mathrm{HbA}_{\mathrm{lc}}$ concentrations was accounted for by the differences between individual children $(p<0 \cdot 001)$. The addition of 'regimen' to the equation accounted for a further $0.4 \%$ of the variance ( $p=N S$ ), while the addition of 'month' to 'child' accounted for a further 3.6\% $(p<0.01)$. There was a small interaction between month and regimen. It can, therefore, be seen that 'month' was a substantially more important predictor of $\mathrm{HbA}_{1 \mathrm{c}}$ concentrations than the differences between the insulin regimens.

Questionnaire. In terms of blood glucose control, nine children preferred three injections, while four preferred two injections. A small majority (seven $v$ five) had felt generally better on the trial regimen. Five children reported more problems with hypoglycaemia on three injections compared with two children on two injections. Thirteen ( $v$ two) found two injections more convenient and 11 ( $v$ five) opted to continue with this regimen.

\section{Discussion}

The options for improving nocturnal control on conventional insulin regimens include delaying the second injection of the day, introducing a third injection, or using an alternative delayed release preparation. ${ }^{11}$ In a previous study, performed on younger children, we found that delaying the second injection of the day until bedtime caused some improvement in morning blood glucose concentrations but higher concentrations in the evening. ${ }^{12}$ This suggested possible benefits from the addition of a third injection before the evening meal. This 
second strategy, of splitting the evening injection, has been used on adults selected on grounds of excess morning glycaemia. ${ }^{7}$

Nine of the 16 children who provided midmorning blood glucose concentrations had their highest concentration of the day at this time. This figure is not quite as high as the incidence of $84 \%$ quoted for adult patients ${ }^{13}$ but, together with examination of the overall blood glucose profiles, suggests that the dawn phenomenon is a frequent and significant finding in this age group.

The three injection regimen caused no overall change in $\mathrm{HbA}_{\mathrm{lc}}$ concentrations and only small changes in blood glucose profiles. The observed blood glucose changes, lower concentrations in the morning and higher concentrations in the middle part of the night, are predictable consequences of delaying the evening intermediate acting insulin. These changes are similar to those found in the study performed on adults, using the same regimen. ${ }^{7}$ It is tempting to suggest that patients selected on the grounds of morning glycaemia might benefit most from the trial regimen. Although the dawn phenomenon was clearly a frequent and important finding in these patients, there was no correlation between the degree of morning glycaemia and the change in $\mathrm{HbA}_{1 \mathrm{c}}$ concentration. Moreover, the adult study, performed on patients showing pronounced morning hyperglycaemia, also failed to alter $\mathrm{HbA}_{1 \mathrm{c}}$ concentrations, despite more substantial changes in blood glucose profiles. ${ }^{7}$

Interestingly, despite the slightly higher blood glucose concentrations seen at 0300 on the three injection regimen, no real change was observed in the incidence of either symptomatic or asymptomatic nocturnal hypoglycaemia. Winter, in a study of unselected diabetic children, found that $18 \%$ suffered asymptomatic hypoglycaemia during one night of continuous blood sampling. ${ }^{14}$ Our incidence of about $10 \%$ of patient nights seems similar, although our patients were monitored much less intensively and at home. None of our patients had symptoms likely to be due to occult hypoglycaemia, but clearly nocturnal hypoglycaemia remains a major obstacle to conventional insulin regimens.

The finding of a seasonal fluctuation in $\mathrm{HbA}_{\mathbf{l c}}$ concentrations might have been due to laboratory changes. The observed changes, however, do not correspond with any known changes in laboratory methodology, staff, or materials. Other workers, reviewing clinic $\mathrm{HbA}_{1 \mathrm{c}}$ results, have reported seasonal changes. Concentrations were lowest in Denmark in midsummer ${ }^{15}$ and in Finland in spring. ${ }^{16}$ Clinic reviews may be subject to the problem of disproportionate patient representation, but our results, in the context of a trial monitoring protocol, show a similar pattern to the Finnish children and suggest an effect of sufficient magnitude to represent a problem for research design. Further examination of this effect is in preparation.

The results of this modest intensification of treatment with insulin were disappointing and warrant comparison with the impressive results reported by others. Schiffrin and colleagues have improved metabolic control in a group of adolescents, who had previously failed to respond to two injections a day, by administering three pre prandial injections with or without a split evening dose. ${ }^{17}$ Their patients admitted to occasionally omitting the inconvenient midday injection but performed at least four blood tests a day, a frequency that, although possibly important for optimal control, ${ }^{18}$ would not have been acceptable to many of our patients. Of the 15 patients approached who declined participation in our study, nine mentioned the blood monitoring protocol as a deterrent, four mentioned the injection regimen, two gave no reason, and two gave other reasons.

Our patients were seen as monthly intervals, and although we encouraged them to contact us for advice between visits, few did so. Intensive medical attention can improve metabolic control, ${ }^{19}$ and more frequent contact and more intensive blood glucose monitoring might have improved metabolic control on both regimens, perhaps particularly if coupled with an insulin dosage adjustment algorithm. Under such conditions, without the 'background noise' of poor control, greater differences between the regimens might become apparent. The results of such a study, however, could not easily be extrapolated to the general clinic setting, where this degree of individual attention is rarely possible. Our experience suggests that intensification of insulin regimens alone is unlikely to improve metabolic control without concurrent intensification of monitoring, particularly during adolescence, an age group renowned for poor and erratic control. ${ }^{8}$

We thank Margaret Evans for performing numerous blood glucose estimations, Dr $J$ Ambler for the $\mathrm{HbA}_{1 \mathrm{c}}$ assays, and Ian Hanning for the $\mathrm{C}$ peptide measurements. Dr Penny Standen gave invaluable statistical advice. FRJH was supported by Novo Laboratories Ltd.

\footnotetext{
References

1 Craig O. Childhood diabetes and its management. 2nd ed. London: Butterworths, 1981.

2 Tchobroutsky G. Relation of diabetic control to development of microvascular complications. Diabetologia 1978;15:143-52.

3 Keen H. Normoglycaemic re-entry and diabetic complications. Diabetic Medicine 1984;1:85-7.

4 Schmidt MI, Hadji-Georgopoulos A, Rendell M, et al. The dawn phenomenon, an early morning glucose rise: implications for diabetic intra-day blood glucose variation. Diabetes Care $1981 ; 4: 579-85$.
} 
5 Campbell PJ, Bolli GB, Cryer PE, Gerich JE. Pathogenesis of the dawn phenomenon in patients with insulin-dependent diabetes mellitus. $N$ Engl J Med 1985;312:1473-9.

${ }^{6}$ Somogyi M. Exacerbation of diabetes by excess insulin action. Am J Med 1959;26:169-91.

${ }^{7}$ Francis AJ, Home PD, Hanning I, et al. Intermediate acting insulin given at bedtime: effect on blood glucose concentrations before and after breakfast. $B r$ Med $J$ 1983;286:1173-6.

${ }^{8}$ Tattersall RB, Lowe J. Diabetes in adolescence. Diabetologia 1981;20:517-23.

${ }^{9}$ Ambler J, Janik B, Walker G. Measurement of glycosylated haemoglobin on cellulose acetate membranes by mobile affinity electrophoresis. Clin Chem 1983;29:340-3.

10 Heding LG. Radioimmunological determination of human Cpeptide in serum. Diabetologia 1975;11:541-8.

1 Turner RC, Phillips MA, Ward EA. Ultralente based insulin regimens-clinical applications, advantages and disadvantages. Acta Med Scand 1983;Suppl 671:75-86.

${ }^{12}$ Hinde FRJ, Johnston DI. Bedtime insulin injections: an alternative regimen. Arch Dis Child 1985;60:311-5.

${ }^{13}$ Francis AJ, Home PD, Walford S, et al. Prevalence of morning hyperglycaemia: determinants of fasting blood glucose concentrations in insulin treated diabetics. Diabetic Medicine 1985;2: 89-94.

14 Winter RJ. Profiles of metabolic control in diabetic children- frequency of asymptomatic nocturnal hypoglycaemia. Metabolism 1981;30:666-72.

15 Mortensen HB, Vestermark S, Kastrup KW. Metabolic control in children with diabetes mellitus assessed by haemoglobin A1c. Acta Paediatr Scand 1982;71:217-22.

16 Karr ML, Akerblom HK, Huttunen NP, et al. Metabolic control in children and adolescents with insulin-dependent diabetes mellitus. Acta Paediatr Scand 1984;73:102-8.

17 Schiffrin A, Desrosiers M, Moffat M, Belmonte MM. Feasibility of strict diabetes control in insulin-dependent adolescents. J Pediatr 1983;103:522-7.

${ }^{18}$ Schiffrin A, Belmonte MM. Multiple daily self-glucose monitoring: its essential role in insulin-dependent patients treated with pump and multiple subcutaneous injections. Diabetes Care 1982;5:479-84.

19 Worth R, Home PD, Johnston DG, et al. Intensive attention improves blood glucose control without further advantage from home blood glucose monitoring: results of a controlled trial. $\mathrm{Br}$ Med $J$ 1982;285:1233-40.

Correspondence to Dr F R J Hinde, Hospital for Sick Children, St Michael's Hill, Bristol BS2 8BJ.

Received 25 October 1985 